\title{
Machine learning: Enhancing the Perception of the Computer
}

\author{
T. Logeswari
}

\begin{abstract}
The advancement of technology and networking allows the use of the Web incredibly important. There is thus an exponential increase in data and information via the Internet. This flow thus is a beneficial field of study which can be defined accurately. Internet traffic detection is a very popular method of identifying information. Although so many methods have been successfully developed for classifying internet traffic, computer training technology among them is most popular. A short study of the classification of Internet traffic on various managed and non-regulated computer teaching systems was undertaken by many researchers. This paper will give various ideas to the other researcher's and help them to learn a lot about machine learning.

Key words:-Machine Learning, artificial intelligence, amazon services.
\end{abstract}

\section{INTRODUCTION}

Machine learning (ML) is a study of algorithms and mathematical models that computer systems use to perform a specific function, based rather on correlations and inferences, without using informative guidance. It is regarded as an artificial intelligence branch. In order to predict and make decisions without needing to directly program the research, Machine Learning Algorithms create a mathematical model centered on sample data, known as training data. The study of machines behaving without being expressly configured is machine learning. In the last decade, auto-driving cars and functional speech recognition, effective web search and an advanced understanding of the human genome have provided us machine learning. Machine learning is today so widespread that, without your understanding, you actually use it dozens of times a day. Most physicists even claim that this is the only way to advance AI on a human level.

\section{MACHINE LEARNING AND ITS TYPES.}

Essentially, algorithms play an important role in machine learning: they understand the trends and can generate solutions on the one side. Algorithms can be subdivided into various categories:

Revised Manuscript Received on April 04, 2020.

* Correspondence Author

Dr.T.Logeswari*, Associate Professor, Dept of Computer Science,New Horizon College,Banglore.Karnansara@gmail.com

(C) The Authors. Published by Blue Eyes Intelligence Engineering and Sciences Publication (BEIESP). This is an open access article under the CC BY-NC-ND license (http://creativecommons.org/licenses/by-nc-nd/4.0/)

\section{Supervised learning}

Sample models are described in advance during supervised learning. To insure that the knowledge is properly distributed to the different model algorithm classes, these must then be defined. That is, on the basis of certain input and output pairs the machine knows. A programmer who functions as a kind of tutor offers the correct values for a certain feedback in the process of supervised learning. The goal is to prepare the machine with different inputs and outputs and links in the sense of successive calculations.

\section{a) Unsupervised learning:}

Artificial intelligence trains through unregulated learning without predefined end goals and without incentives. It is used mainly for segmenting (clustering) instruction. The computer tries to arrange and organize the entered data by certain attributes. A computer, for example, might (very easily) know the coins of different colors, to arrange them, can be sorted according to the stereotypical "color."

b) Partially controlled: Partially monitored schooling is a combination between regulated and unmonitored instruction.

c) Encouraging learning: Through motivation is focused on reward and punishment, just like the traditional training of Skinner. A positive or negative experience informs the program, which responds to a certain circumstance.

d) Active learning: An algorithm enables the search results for unique input data to be focused on predefined questions which are deemed to be relevant within the context of active learning. The algorithm itself usually chooses highly relevant queries.
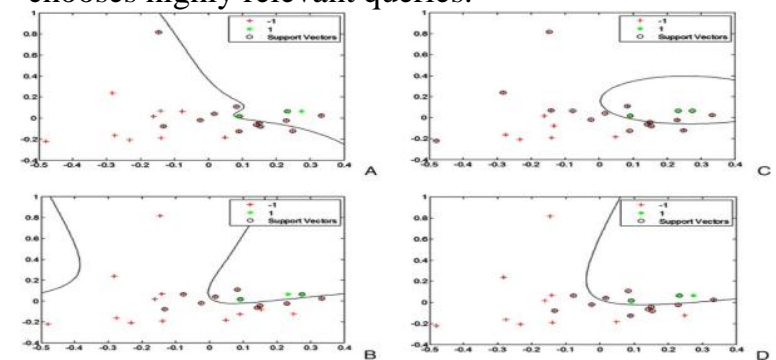

Figure 1:Graphs depicting various SVM

\section{DIFFERENT APPROACHES OF MACHINE LEARNING}

\subsection{Keyword-Based}

In this method we have used the positive/good and negative/bad keyword list and for each review we have counted the number of positive, negative and neutral reviews. The polarity of the highest count is returned by classifier.

$$
\begin{aligned}
& \text { \& Sciences Publication } \\
& \text { (C) Copvriaht: All riahts reserved. }
\end{aligned}
$$

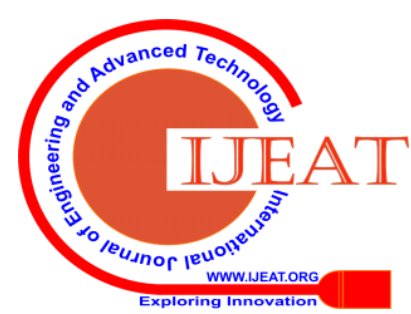




\subsection{Naive Bayes (NB) Classifier}

It is the most basic and most widely employed classification system (1). This classification process calculates / measures the likelihood of a corresponding class based on allocation of terms in text. This model works with the extraction function for BOWs which disregards the word location in the text. To measure the likelihood that the stated function set is part of that class, Bayes Theorem is used. Equation 1 demonstrates that

$\mathrm{P}($ tag $\mid$ Features $) \mathrm{P}=\mathrm{p}($ tag $)+\mathrm{p}($ features $\mid$ tag $)$

\section{$\mathrm{P}($ features $)$}

$\mathrm{P}(\mathrm{tag})$ : The likelihood of id or the choice of random attribute sets is proceeding.

$\mathrm{P}$ (features): The likelihood is preceding the incidence of the stated feature set.

$\mathrm{P}$ (features): The likelihood is followed by the tag defined function collection.

\subsection{Maximum Entropy (ME) Classifiers}

This classificator, which converts labelled functions to vectors with encoding, is also regarded as a conditional exponential classifier. After this, this coded vceptor has been used to approximate the weights of each class, together with the most accountable mark of 1556 Abhilasha Singh Rathor et al. / Procedia Computer Science 132 (2018) 15521561 Abhilasha Singh Rathor / Procedia Computer Science 00 (2018) 000-000 5 Abhilasha Singh Rathor This classifier is a collection of $\mathrm{W}$ \{weights $\}$ parameters that combines joints generated by $\mathrm{E}$ \{encoding\} in the function set. Each $\mathrm{C}\{($ feature set, label $)\}$ pair is mapped to a vector. The calculation below estimates the probability. $\operatorname{gamma}=0$

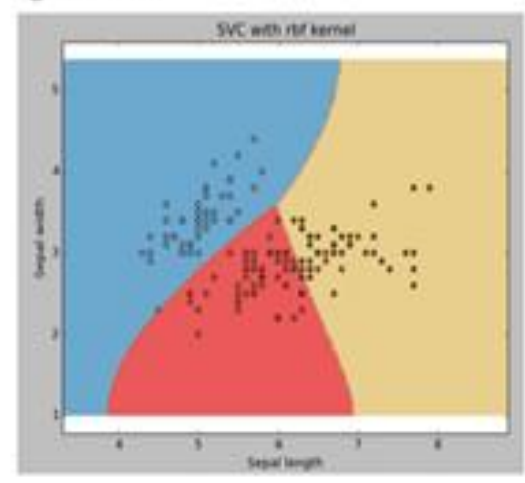

$c=1$

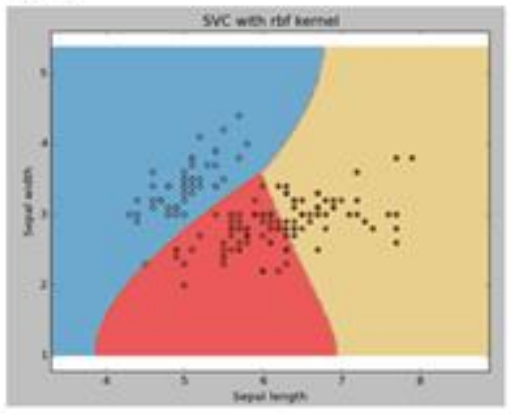

$\operatorname{gamma}=10$

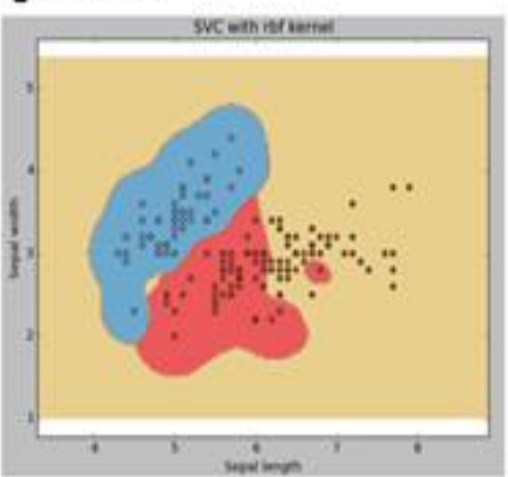

$C=100$

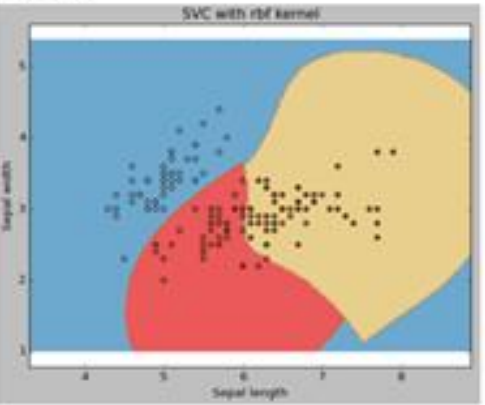

gamma $=100$

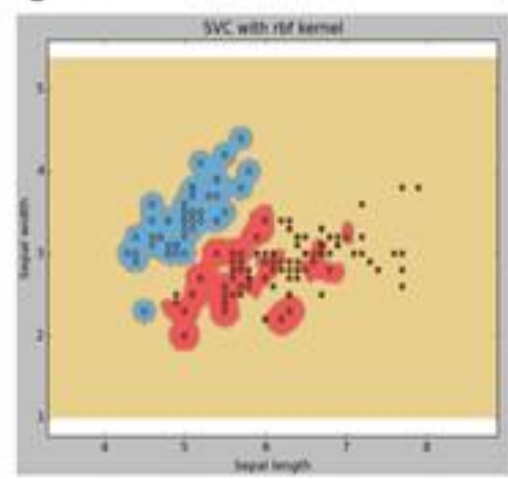

$c=1000$

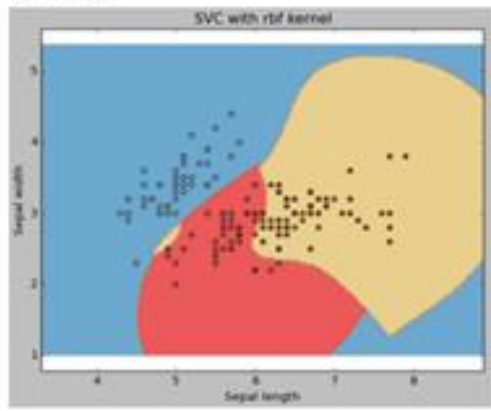

Figure 2: Classifier of Entropy

the weights for interconnection between secret output layers are workable. Input-output mapping feature 1 as follows:

$$
\mathrm{Y}(\mathrm{X})=\sum_{\mathrm{E}=0^{\mathrm{TM}}} \mathrm{Wi} \mathrm{U}(\mathrm{EX}-\mathrm{XiCE})
$$

The RBF network has 3 layer architectures: an entry layer, an opaque layer of non-linear RBF features, and a linear output layer. Radial Basis Function (RBF) is a multi-layer feed system that uses radial baseline functions on each neuron secret layer. A weighted, regular superpolation of all these basis functions is the performance benefit of this RBF neural network. The RBF neural network basic model is shown in Fig. 3. This network addresses weights for interconnection between input and transparent layer, while

Where, U) (hidden layer whish function is applied at each hidden layer neuron, $M$ is centered on the Euclidean distance from the implemented Inputs $\mathrm{X}, \mathrm{Y}(\mathrm{x})$ output mapping function.

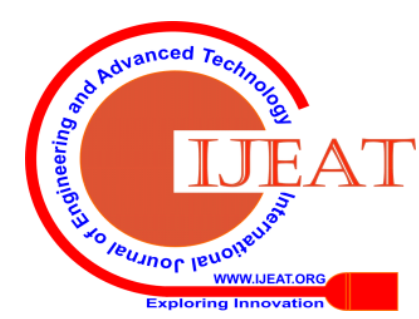




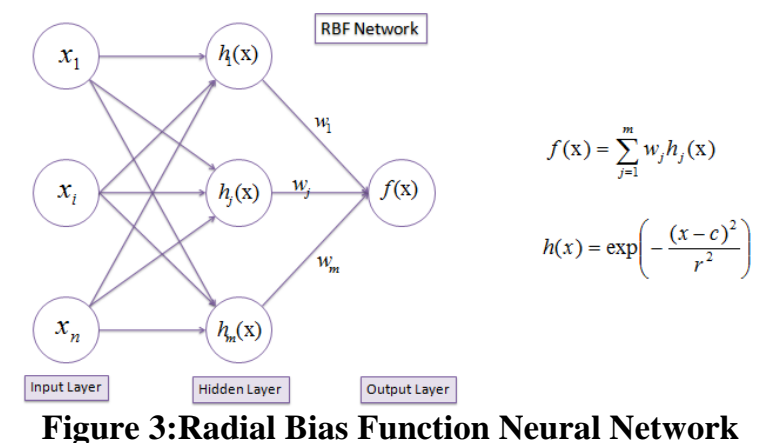

V. APPLICATIONS OF MACHINE LEARNING

Everywhere is Artificial Intelligence. You could use it in one way or another, and you even don't realize it. Machine Learning (ML) is one of AI's common technologies, with apps, devices and computers performing through cognition (much like the human mind). We post several machine learning examples here, which we use everyday, and maybe don't know they are ML powered.

\section{Digital staff support}

Some very common examples of virtual helpers on the internet include Siri, Alexa, Google Now. We help you find details when asked by voice, just as the name suggests. Just trigger them and question, "What is my plan today?"What are German-London routes," or similar issues. Your staff member may look for information and answer your questions or give an order to other services (such as smartphone apps), to gather information. You even can order assistants to do certain activities, such as "Link me to the visa office day after day after morning to schedule an alarm for 6 am next week."

Machine learning is an important element in the processing and refinement of the knowledge based on your previous experience with these staff assistants. Subsequently, this data set is used to provide information customized to your tastes.

In a variety of platforms, robotic assistants are included. Samsung Bixby on Samsung S8 Mobile Apps: Google Allo: For example: Smart Speakers Amazon and Google Home Smartphones

\section{Predictions during transit}

Predictions of Traffic: All of us used GPS navigation. Our existing pages and rates are stored for traffic management on a central server. Such details are used to construct a traffic diagram. Though the issue is that less vehicles are fitted with GPS when stopping traffic or running congestion analyzes. In such cases, machine learning aims to predict where pollution can be identified on the basis of daily experiences.

\section{Tracking videos.}

Picture one person watching many video cameras! Of reality, a hard job to do and also a dull one. This is why it makes sense to have machines prepared for this work.

Today, AI drives the video monitoring system which enables the identification of crime before it occurs. We track people like to sit motionless for a long time, stumble or fall on tables, etc. They imitate odd behavior. It allows the system to warn people, and potentially will help to prevent malfunctions. We help improve monitoring programs when such events are recorded and verified to be valid. This occurs with the history in machine learning.

\section{Products in social media}

Social media plattforms use computer education for their own purposes, from personalizing the news feed to optimizing advertising targeting. Here are a couple of examples to find, use and share on your social media accounts, without knowing they're nothing but ML's applications.

Training in a computer operates on a simple concept: learning through interactions. The contacts you link to, the pages you follow, the passions, job or a community you post, etc., are continuously observed by Facebook.

Training of computers operates on a simple concept: perception of interactions. Facebook often recognizes your contacts, accounts, hobbies, workstations, or communities you share with someone, etc. You are checking very often. A selection of Facebook users, you can become acquainted with, is recommended on the basis of continuing learning. You share the selfie of yours with a buddy and Facebook identifies your person immediately. Face Recognition: Facebook search the locations and predictions on your profile, note the unusual features, then link your friend's list to users. The whole cycle at the back is complex and the accuracy aspect takes care of, but it tends to be a simple implementation of ML at the front end.

Different pins: The core element of computer vision is machine learning, which is a method for extracting valuable information from images and videos. To classify the artifacts (or pins) in the videos, Pinterest uses computer vision and consequently suggests identical pins.

\section{5.Online Detection of Fraud}

Machine learning reveals the potential to make internet environments a safe place and to detect money fraud online. For starters, Paypal uses ML for money laundering security. The organization employs a series of instruments to help them evaluate millions of transactions between buyers and sellers and to discriminate between valid or fraudulent transactions.

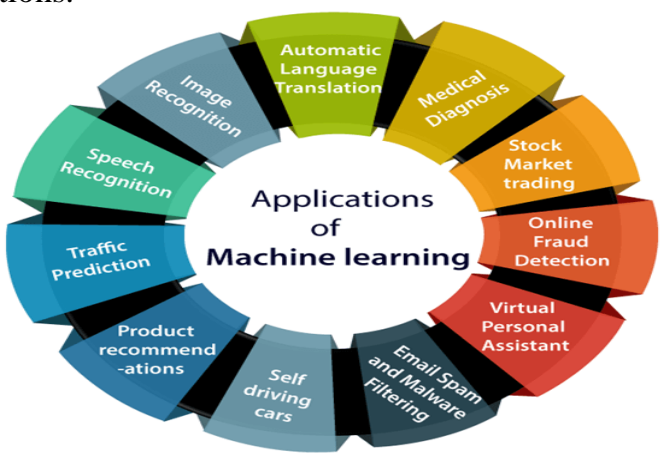

Figure 4 Application of ML

\section{RESULT}

The results show that user reviews are very important for the decision making of customers. But the more surprising and interesting part is to diverge reviews into positive, negative and neutral.

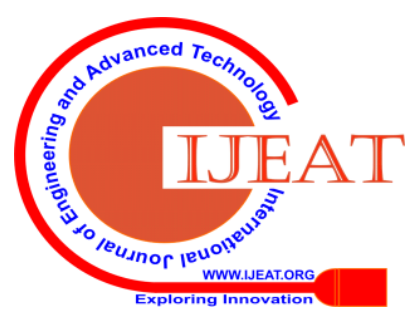


After comparing three machine learning approaches using unigrams features and weighted unigram features in Amazon reviews the accuracy of classifiers is as shown in Table 1.

\begin{tabular}{|c|c|c|c|}
\hline $\begin{array}{l}\text { FEATUR } \\
\text { Es }\end{array}$ & $\begin{array}{l}\text { SUPPORT } \\
\text { VECTOR } \\
\text { MACHINES(S } \\
\text { VM) }\end{array}$ & $\begin{array}{l}\text { MAX } \\
\text { ENTROPY( } \\
\text { ME) }\end{array}$ & $\begin{array}{l}\text { NAÏVE } \\
\text { BAYES(N } \\
\text { B) }\end{array}$ \\
\hline Unigram & 65.42 & 62.84 & 64.96 \\
\hline $\begin{array}{l}\text { Weighted } \\
\text { Unigram }\end{array}$ & 82.36 & 73.5 & 77.89 \\
\hline
\end{tabular}

Unigrams: This is the easiest way to fetch features from a review. We can see from Table 3 that machine learning classifiers had average performance with our feature vector; the reason can be smaller training dataset of 500+ reviews. If the classifier is trained for thousand of reviews than accuracy can improve significantly. Weighted Unigrams: In this we have given weight to the positive/good keywords and negative/bad keywords more than other words when trying to categorize sentiment of review and resulted in economical accuracy as Table 3 shows that. SVM performed finest with $82.36 \%$ accuracy and unexpectedly Naive Bayes (NB) classifier performed better that Max Entropy (ME) i.e. $77.89 \%$ to $73.5 \%$.

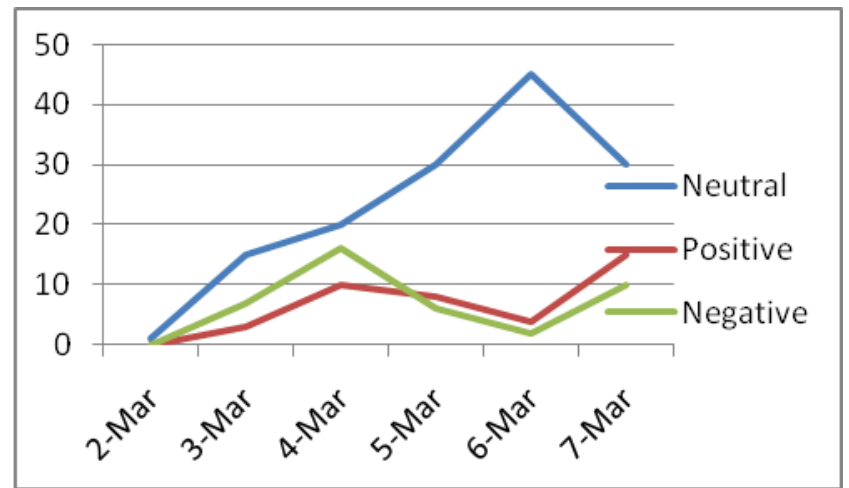
reviews between 2nd to 7 th March.

\section{CONCLUSION}

This research discusses recent progress on the analysis of internet traffic using machine learning techniques. Previous studies in this area demonstrate that machine learning approaches are superior to conventional internet traffic detection techniques. In addition to solving conventional challenges in technology, machine learning often improves its usefulness in this area. Although a range of controlled and unmonitored methods of machine learning had so far been used, the exactness and processing speed were still much better with the expanded data set scale. This is why progress can be made in future research. Product reviews on Amazon are relevant not just to people who buy the product, but also to companies that market it. The study conducted in this report has shown that product reviews not only provide consumers with important information for their purchasing decisions, but also for businesses selling their products. Text comments are very unstructured, so it is first of all important to identify them as good, negative and neutral. For the correct prediction, user reviews must be more effective.

Our analysis has shown that SVM can be used as the foundation for Amazon review, because it is highly accurate.
Figures 5 shows the Amazon review analysis from

\section{REFERENCES}

1. MochamadWahyudi, Dinar AjengKristiyanti. (2016) "Sentiment Analysis Of Smartphone Product Review Using Support Vector Machine Algorithm-Based Particle Swarm Optimization." Journal of Theoretical and Applied Information Technology 91, no. 1.

2. Abhilasha Singh Rathor, Dr. AmitAggarwal, Dr.PreetiDimri.(2017) "Opinion Mining : Insight." International Journal Of Current Engineering And Scientific Research (IJCESR) 4, no. 10

3. Ahlgren, Oskar.(2014) "Research On Sentiment Analysis:The First Decade." Department of Information and Service Economy.

4. AishwaryaBhole, Prof.V.DThombre. (2014) "Review of Sentiment Classification Method and Opinion Mining : The Future Roadmap." International Journal of Engineering Research and Technology (IJERT) 3, no. 3.

5. AkshayAmolik, NiketanJivane, MahavirBhandari, Dr.M.Venkatesan. (2016) "Twitter Sentiment Analysis of Movie." International Journal of Engineering and Technology (IJET) 7, no. 6.

6. AltugAkay, Andrei Dragomir and Bjorn-Erik Erlandsson.(2015) "A Novel Data-Mining Approach Leveraging Social Media to Monitor Consumer Opinion of Sitagliptin." IEEE Journal Of Biomedical And Health Informatics 19, no. 1.

7. Ashish A. Bhalerao, Sachin N. Deshmukh, Sandip D. Mali. (2016) "Predicting Sentiment of User Reviews." International Research Journal of Engineering and Technology (IRJET) 3, no. 5 . [8] BabaljeetKaur, Naveen Kumari. (2016) "A Hybrid Approach to Sentiment Analysis of Technical Article Reviews." I.J. Education and Management Engineering 6, no. 1-11.

8. DONG Shi and et al. The Study of Network Traffic Identification Based on Machine Learning Algorithm; Fourth International Conference on Computational Intelligence and Communication Networks; 2012; IEEE; p. 205-208.

9. Bin $\mathrm{Hu}$ and $\mathrm{Yi}$ Shen. Machine Learning Based Network Traffic Classification: A survey; Journal of Information \& Computational Science; 2012; p. 3161- 3170.

10. LiTing Hu and LiJun Zhang.Real-time Internet Traffic Identification Based on Decision Tree; World Automation Congress (WAC); 2012; IEEE; p. 1-3.

11. Kuldeep Singh and S. Agrawal. Performance Evaluation of Five Machine Learning Algorithms and Three Feature Selection Algorithms for IP Traffic Classification; Evolution in Networks and Computer Communication; ; IJCA; 2011; p. 25-32.

12. IANA, http://www.iana.org/assignments/port-numbers (as of May 2011).

13. Wengang Zhou and et al. Internet Traffic Classification Using Feedforward Neural Network; International Conference on Computational Problem-Solving (ICCP); 2011; IEEE; p. 641-646.

14. Yu Wang and et al. Internet Traffic Classification Using Machine Learning: A Token-based Approach; Fourteenth International Conference on Computational Science and Engineering (CSE); 2011;IEEE; p. 285-289.

15. Murat Soysal and EceGuran Schmidt. Machine learning algorithms for accurate flow-based network traffic classification: Evaluation and comparison; ELSEVIER; 2010; vol. 67; p. 451-467.

16. AbuaglaBabiker and et al. Near Real Time Online Flow-based Internet Traffic Classification Using Machine Learning (C4.5); International journal of engineering (IJE); 2009;

\section{AUTHOR PROFILE}

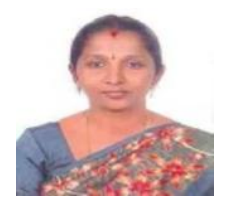

Dr. T.Logeswari is a Associate Professor, Department of Computer Applications, New Horizon College, Bangalore. She obtained her Bachelor's degree in Applied science with Computer Technology from Bharathiar University during 2002, Masters Degree in Computer Applications from Alagappa University during 2006 and M.Phil in Computer Applications from Alagappa University, during 2007. She has completed her $\mathrm{PhD}$ in Computer Science at Mother Teresa Women's University during November 2012. Her specialized areas are Image Processing, Pattern Recognization and Neural Network. Her Ph.D work is focused on Investigations on Intelligent system to detect Brain tumor in the earlier Stage. She has presented many research papers in National, International conferences and Journals.

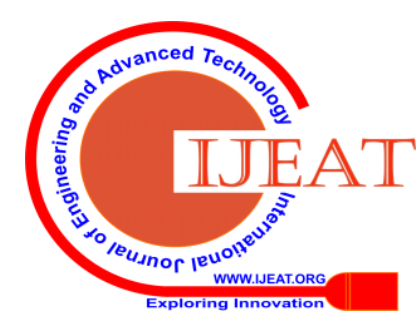

